TERRA. Revista de Desarrollo Local

e-ISSN: 2386-9968

Número 9 (2021), 216-221

DOI 10.7203/terra.9.22075

IIDL - Instituto Interuniversitario de Desarrollo Local

\title{
Reseña. La innovación y sus protagonistas
}

\author{
Aitana Muñoz-Haba \\ Graduada en Sociología y en Ciencias Políticas y de la Administración Pública \\ (Valencia, España) \\ aitanamunozhaba@gmail.com
}

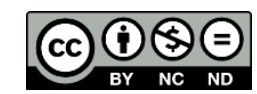

Esta obra se distribuye con la licencia Creative Commons Reconocimiento-NoComercial-SinObraDerivada 4.0 Internacional 


\section{SECCIÓN RESEÑAS}

\section{Reseña. La innovación y sus protagonistas.}

Resumen: En este libro de divulgación se explican las claves de la innovación y sus protagonistas. En la primera parte, los autores se centran en las diferentes claves básicas de la innovación, su historia, definición y clasificaciones. En la segunda, muestran de qué manera la innovación es un fenómeno crucial para el progreso económico. Explican también como el proceso de innovación se produce como un sistema inscrito dentro de su territorio y su contexto sociohistórico. Finalmente, en los apartados finales nos avanzan nuevas perspectivas, y debates y retos más avanzados entorno a esta temática. A lo largo del texto, los autores se esfuerzan en transmitir la importancia del papel de la innovación para, además de generar crecimiento económico, solucionar problemas de la sociedad y el ecosistema.

Palabras clave: Innovación, ciencia, empresa, sistema de innovación, innovación social.

Recibido: 01 de diciembre de 2021

Devuelto para revisión: -

Aceptado: 02 de diciembre de 2021

Referencia / Citation:

Muñoz-Haba, A. (2021). Reseña. La innovación y sus protagonistas. TERRA. Revista de Desarrollo Local, (9), 216-221. DOI 10.7203/terra.9.22075 


\section{Elena Castro Martínez e Ignacio Fernández de Lucio LA INNOVACIÓN Y SUS PROTAGONISTAS}

Madrid (España). Consejo Superior de Investigaciones Científicas (CSIC) y Catarata, 2020. 144 pp.

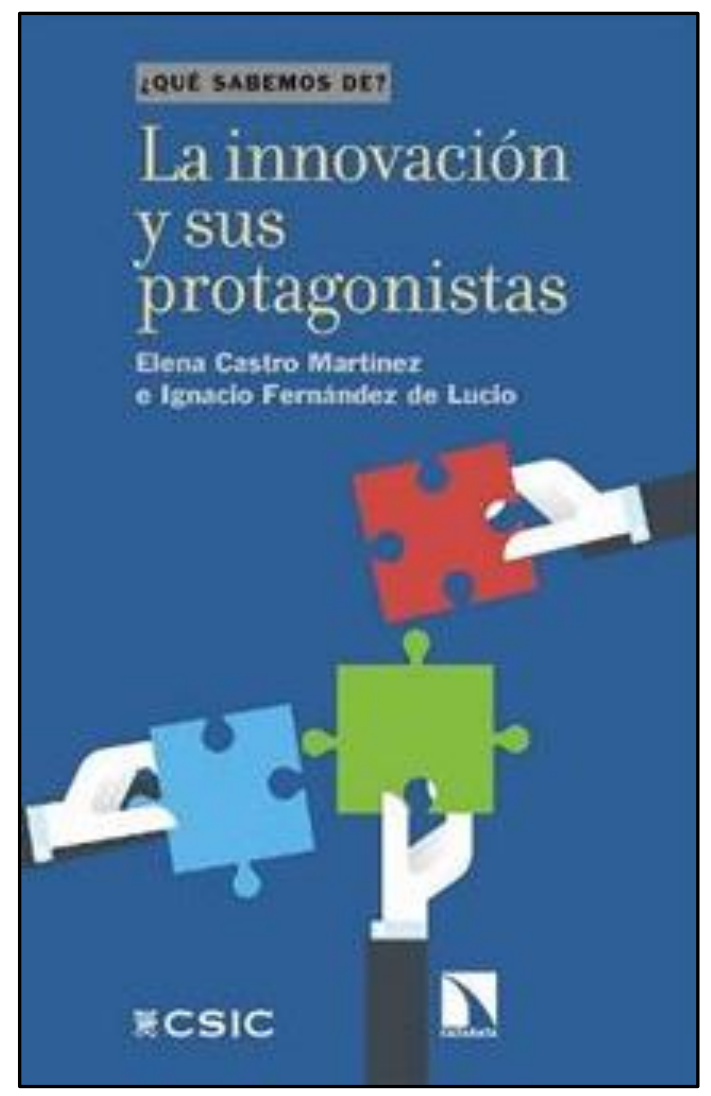

Desde hace unas décadas, la innovación se ha convertido en una de las principales preocupaciones de las empresas, los gobiernos a cualquier escala e incluso de las organizaciones sin ánimo de lucro y con objetivos sociales y medioambientales. No en vano se ha constituido toda una diversidad de centros, departamentos, direcciones oficiales, hubs, fundaciones, etc., tanto de capital público como del privado, dedicados a esta materia. Ante este ferviente interés por la innovación, cabe preguntarnos, como sociedad, qué sabemos realmente sobre este proceso y por qué merece tanta importancia por parte de las personas que dirigen las compañías y las administraciones. Por ello, los investigadores Elena Castro Martínez e Ignacio Fernández de Lucio nos presentan este libro.

"La innovación y sus protagonistas" pertenece a la colección ¿Qué sabemos de? de Catarata, orientada la divulgación científica, dirigida a un público amplio $\mathrm{y}$, definitivamente, no experto. Este sello editorial cuenta con el apoyo del Consejo Superior de Investigaciones Científicas (CSIC). Para el título que presentamos, ambos autores, adscritos a dicha agencia estatal, tienen una larga trayectoria en el estudio del diálogo, no siempre sencillo, entre la academia y su entorno socioeconómico y empresarial, especialmente, en materia de innovación. Y es que, Castro, experta en Ciencias Químicas, y Fernández, ingeniero agrónomo, también han dedicado su carrera a desarrollar todo un corpus de conocimiento sobre la sociología de la ciencia y la tecnología, analizando el papel de éstas en el sistema social y económico en el que se inscriben. Sintetizando toda esta trayectoria, en este libro nos comparten las claves para acercar este proceso de la innovación al público general.

Con prólogo del periodista e historiador Jorge Martínez Reverte y organizada en nueve capítulos, esta obra hace un recorrido por el conocimiento acumulado sobre la innovación y las personas y organismos que la impulsan. Las secciones están estructuradas para responder a las $5 \mathrm{~W}$ (what, who, when, where, why) de la innovación: ¿Qué es la innovación? ¿Quién innova? ¿Cuál es la historia de este fenómeno y de su conceptualización? ¿Cuál es el lugar y el papel de la innovación en el sistema socioeconómico en el que se produce? ¿Por qué es importante entenderla y resolver los debates en torno a ella? Y, además, trata de reflexionar sobre cuáles son las consecuencias, tanto positivas como negativas de esta innovación, es decir, para qué puede servirnos innovar. 
Podríamos considerar que los tres primeros capítulos conforman una primera parte dedicada a presentar las diferentes conceptualizaciones básicas de la innovación, empezando por la historia del concepto (capítulo 1), su definición actual y las diferentes corrientes que la estudian (capítulo 2), así como la diversidad de tipologías y clasificaciones de las innovaciones (capítulo 3). Basada en el Manual de Oslo (2018) y siguiendo la teoría de Schumpeter (1934), esta sección inicial puede ayudar a romper algunos prejuicios sobre este concepto. Por ejemplo, nos recuerdan no todas las innovaciones se basan en el conocimiento científico clásico o analítico, sino que muchas de estas innovaciones en el contexto de ampliación, en la práctica, generando un nuevo conocimiento sintético y basado en la resolución de problemas.

En segundo lugar, el eje central del libro está conformado por los capítulos del 4 al 7. Estos versan sobre el funcionamiento del proceso de innovación (capítulo 5): cómo se genera la innovación, cómo se transfiere y cómo se gestiona. También se detalla cómo la innovación surge de la mano de personas innovadoras (capítulo 7) en el seno de las empresas (capítulo 4), de la academia científica y de otras organizaciones públicas y privadas. En esta segunda parte también se contextualiza este proceso en su entorno socioeconómico y se explica la compleja relación entre la ciencia y la innovación (capítulo 6). Transversalmente, a lo largo de estas secciones, Castro y Fernández exponen los argumentos principales del libro. Básicamente, esta obra presenta la innovación como un fenómeno necesario para la supervivencia de las empresas y las instituciones, y, por tanto, crucial para el progreso económico.

Como vemos, desde la perspectiva de los autores, paradójicamente, solo un sistema que tolera los reajustes y las nuevas soluciones, y, en definitiva, el cambio y la innovación en las maneras de hacer es capaz de mantenerse en el tiempo. Explican como las nuevas corrientes superan el clásico modelo lineal de la innovación y la entienden como un fenómeno abierto, como un Sistema de Innovación inscrito dentro de su territorio y su contexto sociohistórico. Por ejemplo, hablan de la perspectiva de la open innovation de Chesbrough (p. 74). Para completar este argumento se podría haber hecho mención del modelo de la triple hélice de la innovación de Etzkowitz y Leydesdorff (2012), que conceptualiza de manera bastante visual cómo el motor de la innovación está compuesto por las interacciones entre la universidad o academia (primera hélice), la industria o las empresas (segunda hélice) y las administraciones (tercera hélice). Incluso, algunas revisiones de este modelo han incorporado una cuarta y quinta hélice, la sociedad civil y el ecosistema o medio ambiente, respectivamente (Sierra, 2018; Carayannis, 2012).

De este modo, es solo en este sistema interconectado donde surge, sea en una hélice o en otra, una innovación y ésta es rápidamente transferida, incorporada e impulsada por el resto de las hélices. A raíz de esta perspectiva sistémica y relacional, y visto que la innovación tiene un papel importante en el desarrollo del modelo socioeconómico de un territorio, Castro y Fernández hablan de la importancia de la creación de políticas públicas en materia de innovación por parte de las administraciones. Enfatizan el papel de los poderes públicos en la financiación e impulso de las innovaciones, así como en la protección del nuevo conocimiento (patentes, derechos de autor, marcas...).

Finalmente, tras el repaso de los consensos principales que existen en la literatura sobre la innovación, los apartados finales nos avanzan nuevas perspectivas y debates en torno a esta temática. De este modo, los autores invitan al lector a continuar interesándose por este concepto al plantearles retos y cuestiones más avanzadas. El capítulo 8, titulado ¿Cómo nos afectan las innovaciones?, recapitula algunas de las reflexiones avanzadas 
a lo largo del texto y da un salto hacia una perspectiva más macro y más sociológica. Se pregunta por el papel que tiene la innovación en el funcionamiento del sistema económico, y, por tanto, su impacto en el desarrollo económico, tanto positivo como negativo, desde el punto de vista del crecimiento, pero también desde el del mercado laboral y la estructura del empleo. Como vemos, llega a plantearse la propia noción de desarrollo. Personalmente, este capítulo me parece uno de los más interesantes del texto; es de agradecer que en un texto dedicado a la divulgación científica se respete al lector hasta el punto de plantearle que todo lo expuesto como verdades hasta el momento puede ser cuestionable. Plantea que innovación y desarrollo no van automáticamente de la mano. Por eso, el rol de gestión y regulación de las instituciones y la cultura política son cruciales a la hora de orientar la innovación hacia un desarrollo más sostenible y más justo. Es por eso por lo que, a lo largo del libro, dedican a algunas reflexiones al concepto de innovación social, que se refiere a aquellas innovaciones que no se producen con motivaciones de beneficio empresarial, sino que constituyen prácticas o maneras de hacer más justas, y/o más sostenibles que las anteriores. Finalmente, el noveno y último capítulo sirve a modo de conclusión, ya que reflexiona sobre la actual “explosión de la innovación”, es decir, la proliferación de iniciativas innovadoras en el presente y las tendencias futuras.

En general, el lenguaje utilizado por Castro y Fernández es sencillo y la información está esquematizada, sin llegar a ser un manual. Es cierto que un libro de divulgación podría beneficiarse de un mayor uso de elementos gráficos y visuales, como fotos o el resaltado de los conceptos clave. Por otro lado, los ejemplos que nos presentan los autores sirven como ejemplos prácticos, casi anécdotas, que agilizan la lectura y favorecen la comprensión. No obstante, puede que también hagan falta ejemplos más actuales y llamativos para un público más joven. Por ejemplo, un caso paradigmático de empresas que apuestan por una política de innovación siempre presente es el del diseño de las interfaces de redes sociales como Instagram y Facebook. Estas plataformas han llevado un continuo proceso de prueba y error de nuevas e innovadoras funcionalidades (historias diarias, videos cortos, efectos y filtros para las fotos, cambios en el diseño y disposición, nuevas maneras de interactuar con las publicaciones, herramientas de traducción integradas...). Aquello que mueve a estas firmas a innovar son los objetivos de mantener su público objetivo, atraer nuevos clientes e, incluso, aumentar las horas de uso de las aplicaciones, incluso llegando a entrar en conflicto con los límites de la salud de sus usuarios. Por eso, creo que este tipo de ejemplos podrían servir también para exponer las controversias o los límites de poner la innovación y los avances técnicos al servicio de los beneficios empresariales, y cómo estos no tienen por qué coincidir con el interés general de la población o mejorar el bienestar social.

Concluyendo, "La innovación y sus protagonistas", como todo libro dedicado a la divulgación, aporta premisas básicas sobre las teorías sobre la innovación para personas de todo tipo. No obstante, también pone sobre la mesa debates y límites de este concepto y hace algunas reflexiones más sociológicas. Definitivamente, Castro y Fernández abogan por un sistema de innovación con un sector público fuerte, que regule e incentive la innovación, para producir cambios socialmente más justos y sostenibles. Este posicionamiento político podría llegar a producir un cambio en la mentalidad de aquellas personas que decidan interesarse por la innovación por motivos diversos, haciendo que entiendan el papel de la innovación para solucionar problemas de la sociedad y el ecosistema, además de para generar crecimiento económico. 
Aitana Muñoz-Haba

Graduada en Sociología y en Ciencias Políticas y de la Administración Pública

(Universidad de Valencia, Valencia, España)

\section{REFERENCIAS}

Carayannis, E. G., Barth, T. D., and Campbell, D. F. J. (2012). The Quintuple Helix innovation model: global warming as a challenge and driver for innovation. Journal of Innovation and Entrepreneurship, 1(2). https://doi.org/10.1186/2192-5372-1-2

Leydesdorff, L. (2012). The Triple Helix of University-Industry-Government Relations. SSRN Working Paper Series. http://dx.doi.org/10.2139/ssrn.1996760

OCDE/Eurostat (2018). Oslo Manual: Guides for Collecting, Reporting and Using Data on Innovation, $4^{a} \mathrm{ed}$. The Measurement of Scientific, Technological and Innovation Activities, OCDE Publishng/Eurostat, Paris/Luxemburgo.

Schumpeter, J. (1939). The Theory of Economic Development. Harvard University Press, Cambridge [Traducción, Fondo de Cultura Económica, México, 1957].

Sierra, J. (2018). La cuarta hélice y la financiación de la innovación. Journal of Economics, Finance and Administrative Science, 23(45), 128-137. Recuperado de: https://jefas.esan.edu.pe/index.php/jefas/article/view/635 (25/11/2021). 